\title{
L'informatizzazione della cartella clinica
}

\author{
G. Quintaliani, R. Ricci, L. De Megni, U. Buoncristiani
}

UO Nefrologia e Dialisi, Ospedale Silvestrini - Perugia

$\mathbf{L}$

a cartella clinica è lo strumento indispensabile per una corretta gestione dei problemi di qualunque paziente sia esso ambulatoriale che ricoverato. Per la verità una cartella ordinata, ben scritta, continuamente aggiornata e di facile organizzazione rende estremamente agile e sicuro sia l'inquadramento che la gestione di ogni problema clinico connesso al paziente. Tuttavia i requisiti sopra accennati sono difficilmente ottenibili poiché la cartella clinica (e soprattutto la cartella clinica dei pazienti ambulatoriali in dialisi) non è quasi mai ordinata o ben scritta, ancor più raramente, è ben organizzata. Ciò è dovuto essenzialmente al fatto che la massa cartacea cresce con gli anni di trattamento, le varie sezioni si intersecano, i vari esami si disperdono in un mare di altri esami che, seppur simili (ad esempio la busta degli ECG), quasi mai sono ordinati per data.

Vediamo come è organizzata una cartella classica.
L'archivio cartaceo delle cartelle cliniche consiste in un raccoglitore (generalmente organizzato in ordine alfabetico) nel quale sono conservate le cartelle. Ciascuna cartella ha una sua intestazione con $i$ dati anagrafici e contiene tutta la documentazione relativa al paziente. $\mathrm{La}$ cartella "cresce" con il tempo e si arricchisce di documenti che, letti in successione, raccontano la "storia" clinica del paziente, dal primo ingresso fino al suo epilogo.

L'aggiornamento dell'archivio cartaceo non presenta eccessivi problemi: si tratta di raccogliere tutta la documentazione relativa a un paziente in un unico fascicolo che sarà contenuto in uno schedario in un ordine che ne consenta il rapido reperimento. Un limite di tale archivio è l'unicità dei documenti. Se la cartella è fuori posto perché prelevata da qualcuno, non sarà disponibile per la consultazione da parte di altri finché non tornerà al suo posto. Ma il limite maggiore dell'archivio manuale è quello di non poter rispondere a domande, anche semplici, le cui risposte, però, possono rivestire una utilità a volte fondamentale.

Con la cartella clinica cartacea succede più o meno quello che succede con l'elenco telefonico: è facilissimo sapere il numero telefonico $\mathrm{co}$ noscendo ilnome, molto più difficile risulta il contrario oppure sapere il numero degli abitanti di una via, oppure sapere il numero degli utenti il cui nome finisce, ad esempio, con ..ssi (Rossi) e che abitino in una certa via.

In altre parole si fa sempre più sentita l'adozione di strumenti che siano in grado non solo di sapere tutto di una singola cartella (cosa relativamente fattibile anche a mano), ma che siano in grado di "estrarre dati" (magari uno solo come ad esempio l'azotemia) ma da un intero gruppo di cartelle.

Il passaggio da una gestione manuale ad una gestione informatica, sembra essere diventato oggi un passo naturale, quasi obbligatorio anche se a volte si verificano degli eccessi in questo senso, e si infor- 
matizzano anche attività che non ne avrebbero bisogno.

In realtà il confine della convenienza alla informatizzazione quasi mai è una "linea". Molto più spesso è una "fascia" che delimita un'area di "indifferenza", all'interno della quale assumono rilevanza delle motivazioni a volte irrazionali che spingono all'informatizzazione, magari a scapito dell'efficienza.

Non vi è dubbio che l'ingresso di un computer è spesso rivoluzionario. Rivoluziona cioè degli schemi e delle prassi consolidate, introducendo metodi nuovi ai quali a volte è faticoso adattarsi. Ma quanto più il "metodo" informatico è simile a quello manuale e ripropone schemi di lavoro ormai consolidati e collaudati (purché efficienti), tanto più rapido ed accettabile sarà lo sforzo iniziale di apprendimento da parte degli utenti (Fig. 1).

In definitiva, la bontà della soluzione informatica va soprattutto misurata nel rapporto tra dati richiesti e dati restituiti. Quanto più il sistema è in grado di fornire dati aggiuntivi rispetto a quelli immagazzinati, tanto più utile e apprezzabile è la sua adozione. Come esempio banale si può citare l'immissione della data di nascita di un paziente: il computer è in grado di calcolare l'età aggiornata man mano che il tempo passa e quindi di elaborare dati correlati con essa anche se questa non è stata inserita direttamente. Ciò è un esempio banale, ma significativo di quello che si intende quando si afferma che il sistema deve essere strutturato in modo da fornire in uscita (output) più informazioni di quelle di cui è stato fornito.

I dati inseriti debbono prestarsi a successive elaborazioni, anche complesse, in modo da poter restituire informazioni utili agli operatori, per indirizzare il loro lavoro,

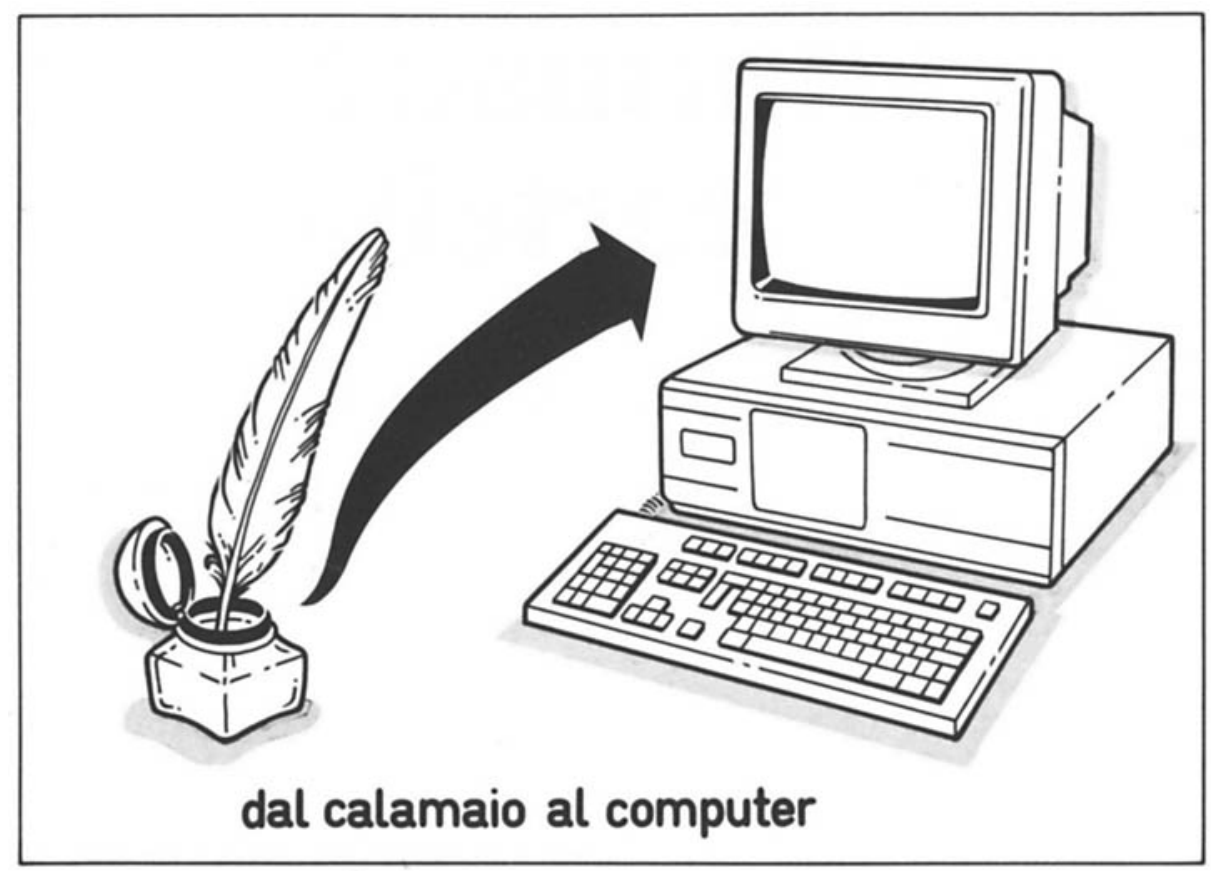

Fig. 1

per far loro prevedere gli andamenti futuri, mettendoli in grado di prepararsi a fronteggiare gli eventi.

Se questo succede, allora il lavoro richiesto dalla informatizzazione del lavoro è ampiamente ricompensato.

L'ingresso del computer deve essere quindi il più "morbido" possibile e deve cercare di riproporre gli schemi di lavoro, ormai collaudati ed efficienti, aggiungendo però delle funzionalità e utilità che giustifichino il suo impiego, i suoi costi e lo sforzo iniziale di apprendimento da parte degli utenti.

Nel caso particolare della informatizzazione di una cartella clinica questi "benefici riflessi", sono abbastanza consistenti e possono ampiamente giustificare lo sforzo iniziale richiesto.

In fondo la sua organizzazione non differisce di molto da quella manuale: è presente uno schedario (archivio o "file") nel quale sono contenuti i dati anagrafici "fissi" dei pazienti, oltre ad un codice identificativo unico per ogni paziente che sarà necessario per identificare qualunque cosa si riferisca a lui. Accanto a questo schedario ne troviamo un altro nel quale vengono conservate le informazioni cliniche identificate da questo codice. Ogni volta che consultiamo una cartella anagrafica, alcuni invisibili "fili" estrarranno automaticamente tutti i "documenti" contrassegnati dallo stesso numero, formando così un fascicolo completo del nominativo estratto, che potrà essere consultato, modificato, aggiornato, stampato, ecc. (Fig. 2).

Naturalmente, se si possono mettere in relazione due differenti archivi, con lo stesso metodo, se ne possono porre in relazione tre, quattro, cinque.

Uno svantaggio del sistema di archivio computerizzato consiste nel fatto che tutte le operazioni di aggiornamento, richiesta, stampa, ecc. vanno effettuate sulla tastiera e che quindi è necessario trovarsi sempre di fronte al computer, ovvero prendere appunti e poi trascriverli. 
TAB. I - REPORT DI UN GRUPPO DI PAZIENTI ENTRATI IN DIALISI DOPO L'1/1/89

\begin{tabular}{|c|c|c|c|}
\hline Nome & Codice nefr. & Tipo dialisi & Dal \\
\hline Alfredo & 20 & CAPD & 12-Sep-89 \\
\hline Angelo & 20 & CAPD & 28-Jun-89 \\
\hline Anna & 00 & CAPD & 8-Jun-89 \\
\hline Antonia & 82 & CAPD & 31-Jan-89 \\
\hline Antonino & 00 & EDO & $11-$ Sep-89 \\
\hline Armando & 10 & EDO & 8-Jun-89 \\
\hline Bruno & 10 & CAPD & 18-Jan-89 \\
\hline Claudia & 10 & EDO & 13-Jul-89 \\
\hline Dante & 00 & EDO & 24-Jun-89 \\
\hline Enzo & 72 & CAPD & 3-Mar-90 \\
\hline Eugenia & 00 & EDO & 9-Nov-89 \\
\hline Fabio & 10 & EDO & 10-Jul-89 \\
\hline Francesco & 82 & CAPD & $30-$ Oct- 89 \\
\hline Giovanni & 50 & EDO & 21-Jul-89 \\
\hline Giuseppe & 40 & CAPD & 22-Dec-89 \\
\hline Giuseppina & 10 & EDO & 13-Jun-89 \\
\hline Italo & 10 & EDO & 17-Feb-90 \\
\hline Lorenzo & 82 & CAPD & 18-Mar-90 \\
\hline Lorenzo & 41 & EDO & 24-Jun-89 \\
\hline Marco & 10 & CAPD & 10-Aug- 89 \\
\hline Maria & 00 & CAPD & 21-Jan-90 \\
\hline Michelina & 80 & CAPD & 5-Apr-89 \\
\hline Natale & 20 & CAPD & 24-Nov-89 \\
\hline Olga & 72 & CAPD & 5-Jan-90 \\
\hline Olimpia & 10 & CAPD & 19-Nov-89 \\
\hline Paola & 41 & CAPD & 22-Feb-89 \\
\hline Paolina & 20 & CAPD & 17-Jan-89 \\
\hline Paolo & 81 & CAPD & 4-Jan-89 \\
\hline Pasqua & 00 & EDO & 10-Jul-89 \\
\hline Pasquale & 20 & EDO & 20-May-89 \\
\hline Pietro & 80 & EDO & 3-Sep-89 \\
\hline Quinto & 20 & CAPD & 1-Mar-89 \\
\hline Raffaele & 10 & EDO & 16-Jan-89 \\
\hline Rita & 10 & CAPD & 24-Mar-90 \\
\hline Ruggero & 25 & EDO & 5-Sep-89 \\
\hline Sante & 20 & EDO & 5-Oct-89 \\
\hline Silvio & 10 & CAPD & 8-Aug-89 \\
\hline Vittorio & 29 & EDO & 9-Mar-89 \\
\hline Wilson & 10 & EDO & 27-Dec-89 \\
\hline Zeffirino & 81 & CAPD & 17-Jun-89 \\
\hline
\end{tabular}

Un altro svantaggio è che alçuni documenti compilati da altri vanno "acquisiti" almeno nei loro dati essenziali (ad esempio i risultati di un'analisi). È quindi necessario un lavoro un po' più lungo e faticoso del semplice inserimento del foglio nel fascicolo. Inoltre alcuni documenti particolari, come radiografie, tracciati ECG, ecc., sono memorizzabili solo mediante costose e sofisticate apparecchiature.

I vantaggi sono peraltro piuttosto consistenti. Innanzi tutto, ciò che è presente nell'archivio può essere presentato sotto varie forme con poco lavoro e in tempi brevíssimi. È possibile ottenere un gran numero di liste o tabulati (report) contenenti le informazioni che servono in quel momento. Avere ad esempio un elenco ordinato per patologia, età e alfabeticamente di tutti pazienti, sarà questione di pochi minuti (Tab. I).

I vantaggi più interessanti si ottengono però nell'elaborazione statistica dei dati contenuti negli archivi. Si potrà sapere immediatamente quanti pazienti sono stati trattati in un certo periodo e fare il confronto con il corrispondente periodo degli
TAB. II

\begin{tabular}{lll}
\hline Tipo & \multicolumn{2}{c}{ Sesso } \\
Trattamento & M & F \\
\hline CAPD & 23 & 34 \\
EDO & 37 & 39 \\
Trap & 23 & 34 \\
\hline
\end{tabular}

anni precedenti, per "vedere" in forma grafica l'andamento dei trattamenti (Tab. II, Figg. 2 e 3). Si potrà analizzare la popolazione dei pazienti in funzione, ad esempio, del sesso, dell'età, dei fattori di 


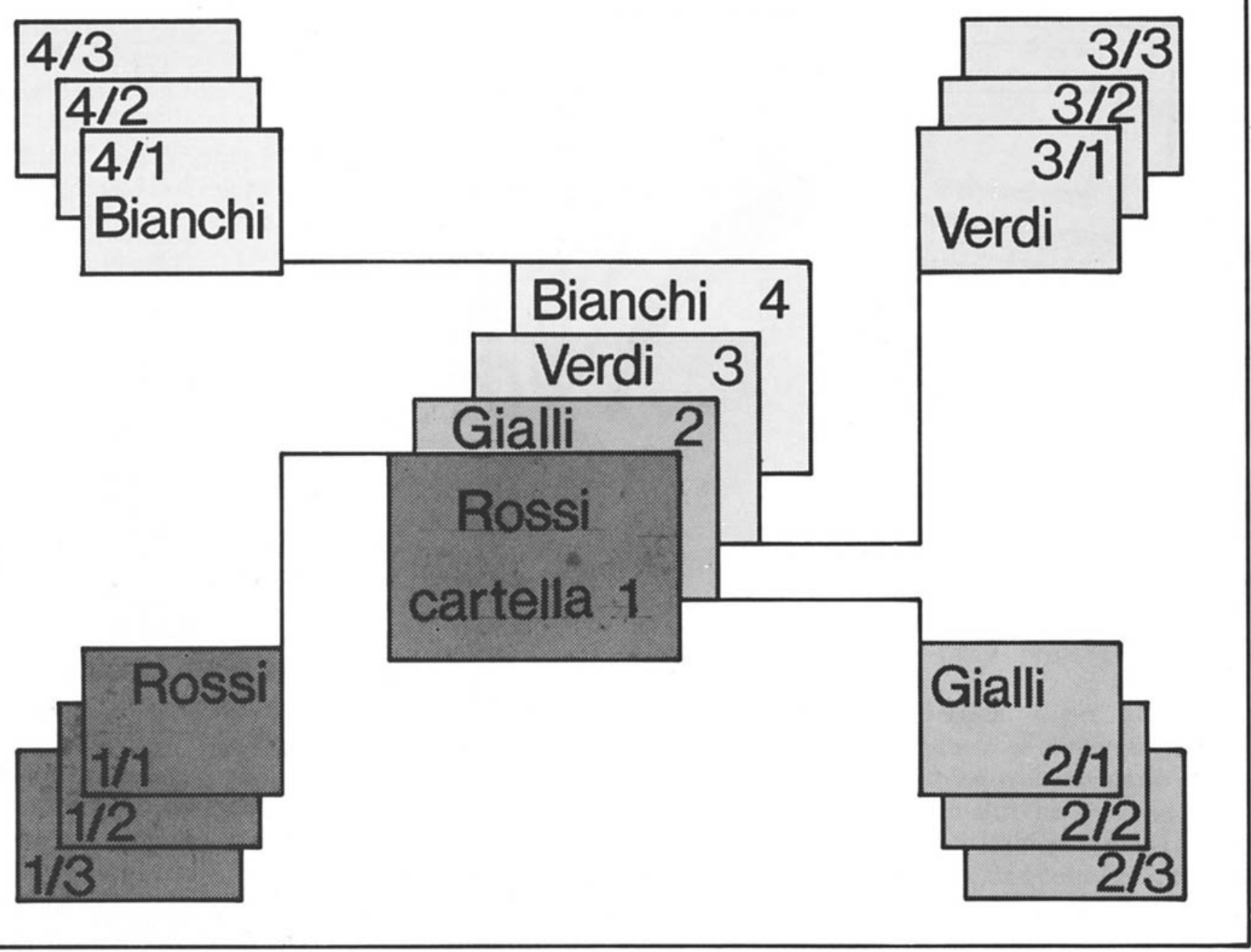

Fig. 2

rischio, delle diagnosi, delle terapie, o di un mixage di queste variabili o di altre presenti nell'archivio. È in questa fase che l'archivio computerizzato restituisce di più di quello che ha ricevuto fornendo informazioni utili alla pianificazione del lavoro, alla ricerca scientifica, alla prevenzione, ecc.

Per ottenere tutto ciò è però indispensabile avere ben chiaro i risultati che si vogliono ottenere, il rapporto costo/benefici tra quello che vorremmo e quello che è possibile ottenere, il tempo per ottenere quello che progettiamo.

È chiaro che alcune soluzioni sono estremamente dispendiose come ad esempio un vero ed efficien-

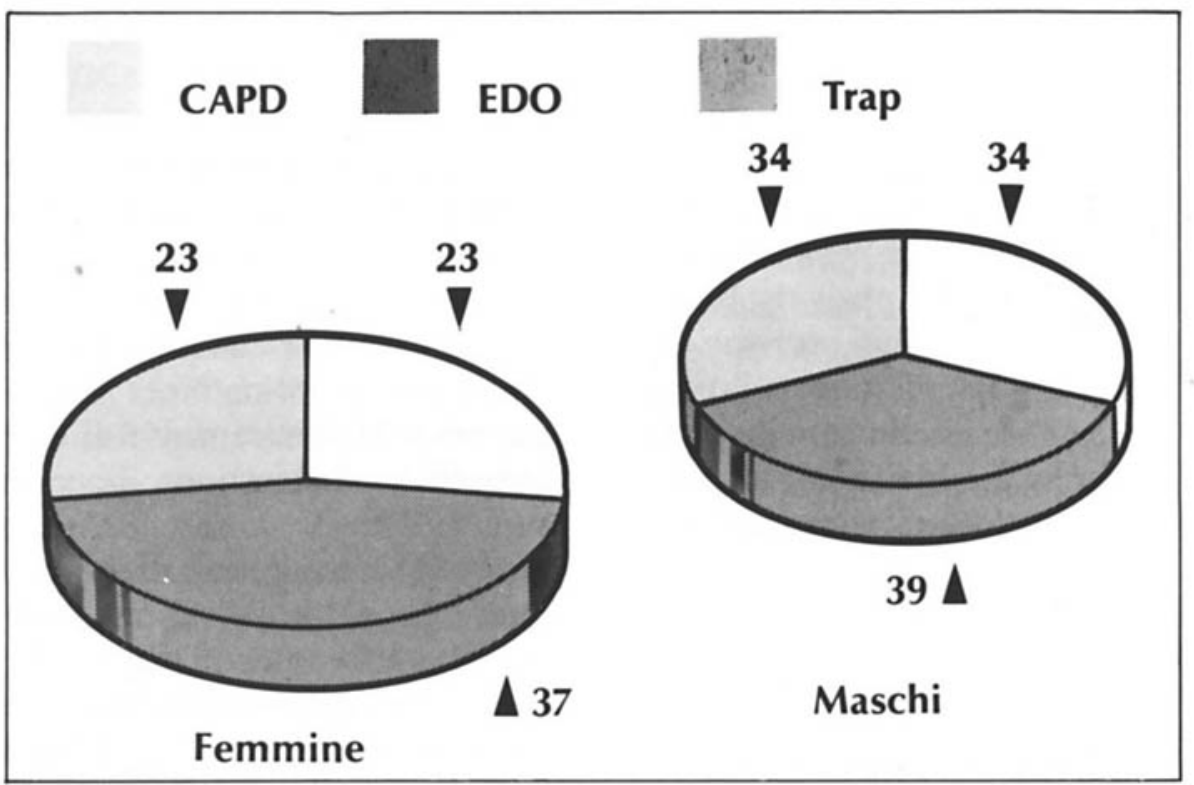

Fig. 3 
te sistema di riproduzione su computer di immagini radiografiche della spesa di svariate decine di milioni, altre estremamente semplici alla portata di qualunque "fai da te" della informatica, come ad esempio una scheda anagrafica di tutti i pazienti.

I punti intermedi sono infiniti e solo l'analisi delle esigenze e delle risorse sia umane che finanziarie fatta $a$ priori può rivelarsi fruttuosa $\mathrm{e}$ non fonte di cocenti delusioni.

A titolo di esempio potremo accennare ad una semplice strutturazione di due archivi: uno anagrafico e l'altro degli esami biochimici.

In altre parole, se vogliamo avere l'andamento di alcuni parametri biochimici nel tempo dei nostri pazienti, dovremo spezzare le informazioni in due. Nell'archivio anagrafico terremo tutte le informazioni fisse del paziente (nome, cognome, data e luogo di nascita, gruppo sangue ecc); nell'archivio esami dovremo invece tenere tutti gli esami che vorremo archiviare con la rispettiva data (è praticamente lo stesso della cartella cartacea con un foglio intestazione e vari fogli esami). In linea di massima dovremo prestare una cura particolare ai parametri da inserire (che in gergo computerese si chiamano campi) poiché dal numero e dalla lunghezza di questi dipende anche la velocità di elaborazione nonché l'occupazione di memoria. Se vorremo avere anche un archivio esami strumentali dovremo collegare al primo anche un terzo archivio. A questo proposito c'è da notare come, in generale, si preferisca usare $i$ codici per la descrizione degli esami strumentali (ad esempio $1=$ ECG normale) e ciò per tre ragioni: una perché si risparmia memoria, due perché i codici si prestano ad elaborazioni statistiche, tre perché si eliminano molti errori sia di battitura che di nomenclatura (anche se quest'ultimo aspetto comporta prima uno sforzo non indifferente per codificare tutte le patologie). L'inconveniente è che spesso non è possibile archiviare sfumature come, ad esempio, ECG normale o ECG nei limiti della norma, ma in genere serve a fare chiarezza, anche nell'ambito dello stesso staff, sulla nomenclatura usata.

Vogliamo a questo punto tracciare alcune considerazioni pratiche sui programmi di archiviazione oggi disponibili e sulla gestione che è possibile ottenere utilizzandoli, nonché di alcuni inconvenienti.

Alcuni programmi di archiviazione sono estremamente facili da usare e non necessitano di programmatore (ad esempio Reflex della Borland). Con questo programma si può archiviare senza problemi un anagrafico del tipo, ad esempio, di quello dell'ANED. Si possono elaborare i dati, graficarli, salvarli e riprenderli in maniera molto semplice e senza conoscere assolutamente nulla di programmazione: tempo di apprendimento circa una settimana. L'inconveniente è che non sono in grado di analizzare grosse quantità di dati (fino a circa $1000-1500$ pazienti) strutturati in un solo archivio, ed inoltre debbono essere affidati a personale che conosca, almeno un minimo, le istruzioni del programma stesso.

Altri programmi sono un po' più difficili da usare (ad esempio DB III o DB IV della Ashon Tate e Pradox della Borland); la loro conoscenza abbisogna di tempi di apprendimento molto più lunghi fino ad arrivare addirittura all'intervento di un programmatore per elaborazioni complesse (dipende ovviamente della sofisticazione del risultato finale che si vuole ottenere). Hanno il grosso vantaggio di poter gestire più archivi contempo- raneamente (ad esempio l'anagrafico e quello dei vari trattamenti nel tempo: tipo EDTA) e di poter essere usati anche da personale con sole conoscenze di dattilografia.

Questi programmi che abbiamo citato (e potrebbero essere infiniti ma abbiamo preferito solo quelli che attualmente sono più diffusi) hanno in comune vantaggi e svantaggi che si possono tradurre nei seguenti punti:

a) costo relativamente ridotto (circa 1-1.5 milioni);

b) possibilità di usarli per innumerevoli archivi diversi (magazzino, anagrafe pazienti, EDTA, studi con protocolli, bibliografia ecc) a patto che si abbia un minimo di dimestichezza con l'informatica ma soprattutto si sia disposti a perdere un po' di tempo sui manuali di istruzione del singolo programma. A questo proposito ricordiamo che i vari corsi sull'uso di computer quasi mai possono aiutare nell'utilizzare questi programmi: è molto più utile spulciare nelle librerie per avere quanti più manuali ed esempi sul programma che intendiamo $u$ sare. Il loro uso può essere poi "espanso" dall'intervento di programmatori professionisti che possono fornire degli eccellenti programmi, molto sofisticati e dedicati al problema oggetto di studio.

Infine esistono programmi dedicati (cioè creati da programmatori per il solo scopo richiesto) che hanno una grossa capacità di elaborazione specifica per il problema che vogliamo affrontare ma che non sono assolutamente in grado di essere utilizzati per scopi diversi; sono in generale più cari (anche alcuni milioni) e difficilmente possono essere adeguati, cambiati o aggiornati senza l'aiuto dello stesso programmatore che li ha creati all'inizio e che quindi diventa automaticamente indispensabile e spesso 
molto caro ad ogni chiamata aggiuntiva. Hanno comunque il pregio di servire proprio a quello che era il progetto originale, di avere diversi blocchi di sicurezza contro problemi accidentali e di rendere automatica qualunque operazione (a patto che sia stata prima prevista) senza alcuna conoscenza di informatica.

Per concludere questa carrellata pratica dobbiamo sottolineare un aspetto che a noi sembra fondamentale: l'interconnessione dei dati. In altre parole è vitale che i dati immagazzinati possano poi essere letti da altri programmi: infatti si verifica spesso che a priori non è sempre facile (neanche con i programmi dedicati) scegliere e programmare $i$ vari tipi di grafici e di analisi statistica.

Esistono a questo scopo svariati programmi che possono graficare e analizzare statisticamente $i$ dati in maniera superba (per i grafici citiamo Chart Microsoft, Harvard Graphics, Polaroid Palette ecc; per la statistica Statgraphic, CSS, SPSS, BMDP e, addirittura, ora è disponibile la versione personal del SAS).

Condizione indispensabile però per l'uso di questi pacchetti è la scambiabilità dei dati che devono essere in formato leggibile a livello internazionale. I formati più diffusi sono ASCII (che significa letteralmente American Standard Code Information Interchange e che è, di fatto, il formato standard e universale per lo scambio di dati), LOTUS e DBF. Molti altri program$\mathrm{mi}$, a loro volta hanno dei sottoprogrammi per convertire i propri dati in questi formati. Ognuno di questi formati è assolutamente interscambiabile con gli altri e assicura una sicura leggibilità anche nel caso in cui si debbano usare i dati senza il programma originale (co- me nel caso di viaggi, di dimostrazioni a congressi, invio a riviste ecc).

Un ultimo accenno velocissimo al problema dell'hardware (cioè del computer). Il consiglio è quello di comperarlo dopo aver scelto il programma da usare ma non solo: il computer è un aggeggio infernale che, cambiando programma, permette di fare le cose più disparate; dal giocare a scacchi a simulare il più difficile esperimento di fisica.

Di solito nei nostri reparti il computer viene usato per: a) scrivere; b) archiviare i dati; c) elaborare i dati sia graficamente che statisticamente. Ognuno di questi aspetti può avere un peso rilevante sulla scelta delle memorie. Il problema memoria merita una precisazione: dividiamo subito la memoria del computer in due tipi la ROM e la RAM. Immaginando di avere una scrivania e un classificatore con le nostre cartelle, la RAM sarà la scrivania e cioè lo spazio a disposizione per depositare e analizzare i dati che ci interessano, mentre invece la ROM sarà il classificatore da cui vengono prese le cartelle per essere depositate nella RAM. Potremo avere un classificatore grande anche come una piazza, ma se avremo una scrivania molto piccola non sapremo dove appoggiare le nostre cartelle a meno di farlo a piccoli gruppi con perdite di tempo e difficoltà di organizzazione. Viceversa avere una grossa scrivania con poche cartelle da appoggiare sarà una inutile spesa aggiuntiva senza grossi risultati. Attualmente il compromesso sembra essere quello di una macchina con processore 286 (meglio ovviamente sarebbe il 386) con memoria RAM di almeno un MB con un disco ROM almeno di $40 \mathrm{MB}$, scheda grafica VGA (sia mono o colore).

Questa configurazione, che attual- mente deve essere considerata come la minima indispensabile (e che quindi lascia fuori tutti i computer tipo XT come ad esempio il glorioso M24 della Olivetti), offre la possibilità di archivi piuttosto grossi con scambi di dati con altri programmi residenti su disco fisso senza la ncessità di continui travasi di dati tra computer e dischetti.

In conclusione l'adozione del computer non deve servire solo a modernizzare esteticamente il lavoro, ma deve essere una scelta cosciente dalla quale ci si deve aspettare un risultato concreto in termini di rapidità, di efficienza, ma soprattutto di conoscenza e analisi dei fenomeni. E da questa tempestiva conoscenza tutti gli operatori attenti potranno trarre indicazioni preziose che li guideranno lungo il cammino non facile, ma denso di soddisfazioni, della analisi decisionale.

Si ringrazia per il supporto tecnico, nonché per la dimostrazione pratica di varie tecniche di archiviazione il Sig. Santini Enrico responsabile della rete Personal Computer della BPS (Banca Popolare Spoleto) 